Canadian

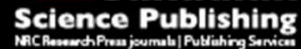

Canadian Journal of Microbiology Revue canadienne de de microbiologie

\title{
Inactivation of Clostridium difficile spore outgrowth by synergistic effects of nisin and lysozyme
}

\begin{tabular}{|c|c|}
\hline Journal: & Canadian Journal of Microbiology \\
\hline Manuscript ID & cjm-2016-0550.R3 \\
\hline Manuscript Type: & Note \\
\hline Date Submitted by the Author: & 01-Feb-2017 \\
\hline Complete List of Authors: & $\begin{array}{l}\text { Chai, Changhoon; Kangwon National University, Division of Applied Animal } \\
\text { Science, College of Animal Life Science } \\
\text { Lee, Kyung-Soo; Kookmin University, Department of Food and Nutrition } \\
\text { Imm, Goo-Sang; Kookmin University, Department of Food and Nutrition } \\
\text { Kim, Young Soon; Korea University, Department of Food and Nutrition } \\
\text { Oh, Se-Wook; Kookmin University, Department of Food and Nutrition }\end{array}$ \\
\hline Keyword: & $<i>$ Clostridium difficile $</ i>$, spore, nisin, lysozyme, synergistic inhibition \\
\hline
\end{tabular}


Title: Inactivation of Clostridium difficile spore outgrowth by synergistic effects of nisin and lysozyme

Changhoon Chai, Kyung-Soo Lee, Goo-Sang Imm, Young Soon Kim, and Se-Wook Oh

C. Chai. Division of Applied Animal Science, Kangwon National University, Chuncheon 24341, Republic of Korea

K. S. Lee, G. S. Imm, and S. W. Oh. Department of Food and Nutrition, Kookmin University, Seoul 02707, Republic of Korea

Y. S. Kim. Department of Food and Nutrition, Korea University, Seoul 02841, Republic of Korea

Corresponding author: Se-Wook Oh (e-mail: swoh@kookmin.ac.kr) 


\begin{abstract}
Inactivating Clostridium difficile spores is difficult, as they are resistant to heat, chemicals, and antimicrobials. However, this note describes inactivation of $C$. difficile spore outgrowth by incubation in a solution containing a germinant $(1 \% \mathrm{w} / \mathrm{v}$ sodium taurocholate $)$, co-germinants $(1 \% \mathrm{w} / \mathrm{v}$ tryptose and $1 \% \mathrm{w} / \mathrm{v} \mathrm{NaCl})$, and natural antimicrobials $(20 \mathrm{nM}$ nisin and $0.2 \mathrm{mM}$ lysozyme). C. difficile spores were resistant to nisin and lysozyme but became susceptible during germination and outgrowth triggered and promoted by sodium taurocholate, tryptose, and $\mathrm{NaCl}$. The degree of inactivation of germinated and outgrowing $C$. difficile spores by both nisin and lysozyme was greater than the sum of that by nisin and lysozyme individually, suggesting synergistic inactivation of $C$. difficile spores. The germinant, co-germinants, and natural antimicrobials used in this study are safe for human contact and consumption. Therefore, these findings will facilitate the development of a safe and effective method to inactivate $C$. difficile spore.
\end{abstract}

Keywords: Clostridium difficile, lysozyme, nisin, spore, synergistic inactivation 
Clostridium difficile, a Gram-positive and spore-forming obligate anaerobe, is the causative agent of $C$. difficile-associated colitis (CDAC), which manifests as clinical symptoms ranging from asymptomatic intestinal colonization to diarrhea, colitis, pseudomembranous colitis, and death (Ghose 2013). C. difficile is acquired nosocomially, and the organism is resistant to multiple antibiotics (Carroll and Bartlett 2011). C. difficile enters a dormant spore state in environments unfavorable for survival. $C$. difficile spores are commonly found on bed commodes and stainless steel surfaces previously occupied by $C$. difficile-infected patients (Weber et al. 2010). Transmission of $C$. difficile is generally mediated via contact with surfaces in healthcare facilities (including rooms, beds, bed commodes, rectal thermometers, and medical devices) used by $C$. difficile-infected patients, particularly in hospitals (Weber et al. 2010). Thus, removing $C$. difficile spores on contaminated surfaces is crucial for preventing transmission of C. difficile (Weber et al. 2010).

Nisin, a peptide antibiotic with activity against Gram-positive bacteria, effectively inhibits $C$. difficile; indeed, its in-vitro activity against $C$. difficile in the vegetative form is eightfold greater than those of vancomycin and metronidazole, primary antibiotics used for the treatment of CDAC (Bartoloni et al. 2004). Nisin is a candidate agent for the treatment of CDAC (Ali et al. 2013). A synergistic effect of the nisin and lysozyme combination on the inhibition of vegetative C. difficile has been reported (Chai et al. 2015). However, inactivating C. difficile spores is difficult because of their thick polymer cortex and outer proteinaceous coat, which render the spores resistant to heat, chemicals, and antimicrobials (Permpoonpattana et al. 2011).

C. difficile spores in the intestines germinate and outgrow to vegetative cells in response to germination triggers (germinants), primarily bile salts and specific amino acids, particularly if the gut microbiome is disrupted by administration of antibiotics (Poutanen and Simor 2004; 
Theriot and Young 2015; Wilson 1983). C. difficile spores can be induced to germinate and outgrow in-vitro by sodium taurocholate and glycine or histidine (Ramirez et al. 2010; Sorg and Sonenshein 2008). C. difficile spores during germination and outgrowth are susceptible to ultraviolet (UV) irradiation and nisin (Nerandzic and Donskey 2013). Induction of spore germination and outgrowth and inactivation of the spores using non-toxic substances may be a practical strategy for the development of a liquid surface sanitizer to reduce the $C$. difficile spore burden in healthcare facilities and on the skin of healthcare workers.

This note describes the induction of germination and outgrowth of $C$. difficile spore as well as effective inactivation of the spore using substances that are safe for human contact and consumption. Germination of $C$. difficile spores is triggered by sodium taurocholate and promoted by specific amino acids (Nerandzic and Donskey 2013; Ramirez et al. 2010; Sorg and Sonenshein 2008). However, purified amino acids were used in previous investigations of $C$. difficile spore germination. The high cost of purified amino acids has hampered the development of a method to inactivate $C$. difficile spores that can be applied in healthcare facilities. We evaluated the ability of protein hydrolysates (peptone, yeast extract, tryptone, and tryptose) to promote spore germination and outgrowth. We also investigated the effects of $\mathrm{NaCl}$ concentration on the germination and outgrowth of $C$. difficile spores triggered by sodium taurocholate, as it has been reported that osmotic stress affects the germination of bacterial spores, including those of Bacillus and Clostridium species (Nerandzic and Donskey 2013; Preston and Douthit 1984). The findings of the aforementioned investigations were applied to formulate a solution that would induce germination and outgrowth of $C$. difficile spores (GO solution). Inactivation of $C$. difficile spore outgrowth by nisin and lysozyme was investigated by enumerating $C$. difficile spores surviving in GO solution containing nisin and lysozyme. 
C. difficile ATCC 9689 (PCR ribotype 001) was purchased from the Korean Collection for Type Cultures and cultured overnight in brain-heart infusion broths (BHI broth; OXOID) at $37^{\circ} \mathrm{C}$ under anaerobic conditions $\left(80 \% \mathrm{~N}_{2}, 10 \% \mathrm{H}_{2}\right.$, and $\left.10 \% \mathrm{CO}_{2}\right)$ in an anaerobic chamber (Don Whitley Scientific). For sporulation, $1 \mathrm{~mL}$ of freshly prepared C. difficile ATCC 9689 culture was added to each of $6 \mathrm{BHI}$ agar plates and incubated for 4 days at $37^{\circ} \mathrm{C}$ under anaerobic conditions. C. difficile spores were collected by flooding the plates with $10 \mathrm{~mL}$ of ice-cold distilled water (DW). C. difficile spores collected in $15 \mathrm{~mL}$ centrifuge tubes were stored overnight at $4^{\circ} \mathrm{C}$. The spore suspension was centrifuged at $4000 \times g$, supernatant was discarded, and spore pellets were resuspended in $10 \mathrm{~mL}$ of ice-cold DW. This was repeated three times, and then the spore suspension was stored at $4^{\circ} \mathrm{C}$. Media used to culture $C$. difficile and prepare $C$. difficile spores were maintained under anaerobic conditions at least overnight. The spore suspension was heated at $60^{\circ} \mathrm{C}$ for $20 \mathrm{~min}$ to inactivate vegetative $C$. difficile prior to use.

Solutions composed of $1 \% \mathrm{w} / \mathrm{v}$ sodium taurocholate (Sigma-Aldrich) with various amendments were prepared in DW and then inoculated with $C$. difficile spores. These amendments were: 1) 1\% w/v protein hydrolysate [peptone (Bacteriological Peptone L37; Oxoid), yeast extract (Yeast Extract L21; Oxoid), tryptone (Tryptone L42; Oxoid) or tryptose (Tryptose L47)]; 2) $0.2,0.4,0.6,0.8$, or $1.0 \% \mathrm{w} / \mathrm{v}$ tryptose; 3 ) $0.1 \% \mathrm{w} / \mathrm{v}$ tryptose as well as 0.25 , $0.5,1,5,10$, or $15 \% \mathrm{w} / \mathrm{v} \mathrm{NaCl}$; and 4$) 0.2,0.4,0.6,0.8$, or $1.0 \% \mathrm{w} / \mathrm{v}$ tryptose along with $1 \% \mathrm{w} / \mathrm{v}$ $\mathrm{NaCl}$; see Fig. 1A, B, C, and D, respectively. Twenty milliliter of the solution inoculated with $C$. difficile spores was placed in a 50-mL beaker, and incubated for $2 \mathrm{~h}$ under an aerobic atmosphere at room temperature to explore the possibility of enhancing germination and outgrowth of $C$. difficile spores under ambient environmental conditions. After incubation for $2 \mathrm{~h}, 1 \mathrm{~mL}$ of the solution was transferred to a $1.5 \mathrm{~mL}$ microtube and centrifuged at $10000 \times \mathrm{g}$ for $2 \mathrm{~min}$ to collect 
spores. Collected spores were resuspended in $1 \mathrm{~mL} \mathrm{DW}$, heated at $60^{\circ} \mathrm{C}$ for $20 \mathrm{~min}$, diluted with $0.1 \%$ peptone water, and plated on TBHI agar [BHI agar containing $5 \mathrm{mg} \mathrm{mL}^{-1}$ yeast extract (Oxoid), 0.1\% L-cysteine (Sigma-Aldrich), and 0.1\% sodium taurocholate (Sigma-Aldrich)]. The TBHI agar plates were incubated for $24 \mathrm{~h}$ at $37^{\circ} \mathrm{C}$ under anaerobic conditions, and then $C$. difficile spores were counted $(N)$. The logarithmic difference between $N$ at $0 \mathrm{~min}\left(N_{0}\right)$ and at $2 \mathrm{~h}$ $\left(N_{2 h}\right)$ was regarded as the level of germinated and outgrowing $C$. difficile spores $(L O$; $\left.L O=\log N_{0}-\log N_{2 h}\right)$. Because the logarithmic value of $N_{0}$ varied from 4 to $5 \log \mathrm{CFU} \mathrm{mL} \mathrm{m}^{-1}$, the $L O$ was divided by the logarithmic value of $N_{0}$, and the relative level of $L O(R L O$; $R L O=L O / \log N_{0}$ ) was used to evaluate the effects of protein hydrolysates on the germination and outgrowth of $C$. difficile spores and to formulate the GO solution.

Inactivation of $C$. difficile spore outgrowth by nisin and lysozyme was investigated by enumerating surviving $C$. difficile spores during incubation in GO solution containing nisin and lysozyme. Solutions composed of $1 \% \mathrm{w} / \mathrm{v}$ sodium taurocholate, $1 \%$ tryptose, and $1 \% \mathrm{NaCl}$ with various amendments were prepared in DW. These amendments were: 1) 20, 40, 60, 80, or 100 $\mathrm{nM}$ nisin (nisin A; Sigma-Aldrich); 2) 0.2, 0.4, 0.6, 0.8, or $1.0 \mathrm{mM}$ lysozyme (Sigma-Aldrich); and 3) $0.2 \mathrm{mM}$ lysozyme, and 5, 10, 15, or $20 \mathrm{nM}$ nisin; see Figs. 1, 2, and 3, respectively. Twenty milliliter of the solution inoculated with $C$. difficile spores was placed in a 50-mL beaker and incubated for $2 \mathrm{~h}$ under aerobic conditions at room temperature. During the incubation, $1 \mathrm{~mL}$ of the solution was transferred to a $1.5 \mathrm{~mL}$ microtube every $30 \mathrm{~min}$ and centrifuged at $10000 \times \mathrm{g}$ for 2 min. Pelleted $C$. difficile spores were resuspended in $1 \mathrm{~mL}$ of DW to wash out nisin and lysozyme and centrifuged at $10000 \times \mathrm{g}$ for $2 \mathrm{~min}$; this procedure was repeated twice. Collected $C$. difficile spores were resuspended in $1 \mathrm{~mL} \mathrm{DW}$ and cultured on TBHI agar plates, and surviving C. difficile spores $\left(N^{s}\right)$ were counted after incubating the TBHI agar plates for $24 \mathrm{~h}$ at $37^{\circ} \mathrm{C}$ 
under anaerobic conditions. The logarithmic value of $N^{s}$ in GO solution containing nisin or lysozyme or both nisin and lysozyme at $0 \min \left(\log N_{0}^{s}\right)$ varied from 4 to $5 \log C F U \mathrm{~mL}^{-1}$. Hence, the logarithmic value of $N^{s}$ at a certain time $\left(\log N_{t}^{s}\right)$ was divided by $\log N_{0}^{s}$, which represented the relative level of surviving spores $\left(R L S ; R L S=\log N_{t}^{s} / \log N_{0}^{s}\right)$. All tests described above were carried out in triplicate, and the results are presented as means and standard deviations. The germination and outgrowth of $C$. difficile spores data were subjected to one-way analysis of variance, and $\mathrm{P}$-values $<0.05$ were considered to indicate significance.

Sodium taurocholate alone did not induce germination and outgrowth of $C$. difficile spores; another carbon source, such as protein hydrolysate, was required (Fig. 1A). C. difficile spores exposed to sodium taurocholate responded differently to peptone, yeast extract, tryptone, and tryptose (Fig. 1A). A greater number of $C$. difficile spores germinated and outgrew in the presence of $1 \% \mathrm{w} / \mathrm{v}$ sodium taurocholate and $1 \% \mathrm{w} / \mathrm{v}$ tryptose than in the presence of the other protein hydrolysates, as indicated by the greater $R L O$ value (Fig. 1A). According to the manufacturer's product information, peptone and tryptose were derived from enzymatic hydrolysis of meat, tryptone from enzymatic hydrolysis of milk protein, and yeast extract from yeast. Thus the amino acid composition of each protein hydrolysate is different. Tryptose contains abundant glycine and alanine compared to peptone, yeast extract, and tryptone (manufacturer's product information). Alanine and glycine act as co-germinants for $C$. difficile spores and promote $C$. difficile spore germination more effectively than any other amino acids (Howerton et al. 2011). Therefore, the greater $R L O$ values observed in the presence of tryptose relative to other protein hydrolysates might be due to the greater abundance of glycine and alanine in this hydrolysate (Fig. 1A). The $R L O$ value increased as the tryptose concentration increased from 0 to $0.8 \% \mathrm{w} / \mathrm{v}$, but there was no increment in the $R L O$ value as the tryptose 
concentration increased from 0.8 to $1.0 \% \mathrm{w} / \mathrm{v}$ (Fig. 1B). The germination and outgrowth of $C$. difficile spores triggered and promoted by sodium taurocholate and tryptose were stimulated by $\mathrm{NaCl}$ (Fig. 1C). The $R L O$ value increased with the $\mathrm{NaCl}$ concentration from 0 to $1 \% \mathrm{w} / \mathrm{v}$ in the presence of $1 \% \mathrm{w} / \mathrm{v}$ sodium taurocholate and $0.1 \% \mathrm{w} / \mathrm{v}$ tryptose (Fig. 1C). However, the $R L O$ values decreased as the $\mathrm{NaCl}$ concentration increased from 1 to $15 \% \mathrm{w} / \mathrm{v}$ (Fig. 1C). In the presence of $1 \% \mathrm{w} / \mathrm{v}$ sodium taurocholate and $1 \% \mathrm{w} / \mathrm{v} \mathrm{NaCl}, C$. difficile spore germination and outgrowth increased as the tryptose concentration increased from 0 to $0.8 \% \mathrm{w} / \mathrm{v}$ (Fig. 1D). Indeed, all $C$. difficile spores germinated and outgrew in solutions containing $1 \% \mathrm{w} / \mathrm{v}$ sodium taurocholate, $1 \% \mathrm{w} / \mathrm{v} \mathrm{NaCl}$, and 0.8 and $1.0 \% \mathrm{w} / \mathrm{v}$ tryptose, as indicated by an $R L O$ value of 1 (Fig. 1D). These $R L O$ values (Fig. 1) led to the formulation of a GO solution composed of $1 \%$ $\mathrm{w} / \mathrm{v}$ sodium taurocholate, $1 \%$ tryptose, and $1 \% \mathrm{NaCl}$ in $\mathrm{DW}$.

C. difficile in a vegetative state is susceptible to nisin and lysozyme, the combination of which exerts synergistic effects (Chai et al. 2015). C. difficile spores were not inactivated by either nisin or lysozyme if these treatments were used without GO solution (data not shown). However, $C$. difficile spores that were germinated and outgrew in GO solution were susceptible to nisin and lysozyme, which resulted in the decrease in $R L S$ values (Fig. 2A and B). Inactivation of $C$. difficile spore outgrowth by nisin and lysozyme was dependent on their concentrations; the $R L S$ values decreased with increasing nisin and lysozyme concentrations (Fig. 2A and B). Germinated and outgrowing $C$. difficile spores were likely more sensitive to nisin than to lysozyme, as inactivation occurred at a lower concentration of nisin than of lysozyme. After incubation for 2 $\mathrm{h}$, the $R L S$ values of $100 \mathrm{nM}$ nisin and $1 \mathrm{mM}$ lysozyme were 0.12 and 0.13 , respectively (Fig. 2A and B). The difference between the dose of nisin and that of lysozyme required to inactivate $C$. difficile spore outgrowth might be due to differences in their antimicrobial mechanisms. Both 
nisin and lysozyme exhibit antimicrobial activity, especially toward Gram-positive bacteria, including Clostridium spp. (Chai et al. 2015; Chung and Hancock 2000). Nisin is transported across the peptidoglycan layer and incorporated into the cytoplasmic membrane of Grampositive bacteria, resulting in leakage of cytoplasmic contents (AlKhatib et al. 2014). In contrast, lysozyme hydrolyzes the glycosidic bonds between $\mathrm{N}$-acetylglucosamine (NAG) and $\mathrm{N}$ acetylmuramic acid (NAM) in the peptidoglycan layer encasing the cytoplasmic membrane of Gram-positive bacteria, resulting in lysis (Kirby 2001).

C. difficile spore outgrowth was synergistically inactivated by the combination of nisin and lysozyme (Fig. 2C). The decreases in the $R L S$ values of the $0.2 \mathrm{mM}$ lysozyme and $20 \mathrm{nM}$ nisin treatments after $2 \mathrm{~h}$ of incubation were only 0.06 and 0.21 , respectively $(R L S$ values, 0.94 and 0.79 , respectively) (Fig. 2A and B). However, $R L S$ values were markedly lower in the presence of both lysozyme and nisin in GO solution than in the presence of only lysozyme and nisin (Fig. 2C). Notably, no viable $C$. difficile spores were detected after $2 \mathrm{~h}$ of incubation in GO solution containing both $20 \mathrm{nM}$ nisin and $0.2 \mathrm{mM}$ lysozyme ( $R L S$ value, 0$)$ (Fig. 2C). The decrease in the $R L S$ value after $2 \mathrm{~h}$ of incubation of $C$. difficile spores in GO solution containing $20 \mathrm{nM}$ nisin and $0.2 \mathrm{mM}$ lysozyme was greater than the sum of the decrease in the $R L S$ values in the presence of $20 \mathrm{nM}$ nisin and $0.2 \mathrm{mM}$ lysozyme alone (Fig. 2C). Lysozyme likely hydrolyzes the NAGNAM linkages of the peptidoglycan layer of $C$. difficile spores that were germinated and outgrew in GO solution, which possibly facilitated the transport of nisin across the peptidoglycan layer to the cytoplasmic membrane, thus resulting in a synergistic effect on $C$. difficile spore outgrowth. Synergistic antimicrobial effects of the nisin and lysozyme combination on pathogens in food and on food packaging have been reported by others (Bhatia and Bharti 2015; Lucera et al. 2012). 
In this study, $C$. difficile spores were inactivated by a solution containing $1 \% \mathrm{w} / \mathrm{v}$ sodium taurocholate, $1 \% \mathrm{w} / \mathrm{v}$ tryptose, $1 \% \mathrm{w} / \mathrm{v} \mathrm{NaCl}, 20 \mathrm{nM}$ nisin, and $0.2 \mathrm{mM}$ lysozyme, without additional treatment. Because sodium taurocholate, tryptose, $\mathrm{NaCl}$, nisin, and lysozyme have been proven safe for human consumption (United States. Food and Drug Administration 2016), this note suggests the feasibility of developing a safe and effective method of reducing the $C$. difficile spore burden. However, there should follow a study for the inactivation of $C$. difficile spore deposited on actual surfaces of healthcare facilities using the GO solution containing nisin and lysozyme.

\section{Acknowledgements}

This study was supported by a program of High Value-added Food Technology Development of Korea Institute of Planning and Evaluation for Technology in Food, Agriculture, Forestry and Fisheries (IPET) funded by the Ministry of Agriculture, Food and Rural Affairs (MAFRA) (Grant No. 313032-03-2-HD020). This study was also supported by 2016 Research Grant from Kangwon National University.

\section{References}

Ali, A.H., Hale, T.W., Khasawneh, F.A., Urban, R.S., Werner, H.V., and Smalligan, R.D. 2013. Nisin and Clostridium difficile: A potentially effective treatment for an increasingly problematic disease. The American Journal of Gastroenterology 108(4): 625-625.

AlKhatib, Z., Lagedroste, M., Fey, I., Kleinschrodt, D., Abts, A., and Smits, S.H.J. 2014.

Lantibiotic immunity: Inhibition of nisin mediated pore formation by NisI. PLoS ONE 9(7): e102246. doi: 10.1371/journal.pone.0102246. 
Bartoloni, A., Mantella, A., Goldstein, B.P., Dei, R., Benedetti, M., Sbaragli, S., and Paradisi, F. 2004. In-vitro activity of nisin against clinical isolates of Clostridium difficile. Journal of Chemotherapy 16(2): 119-121. doi: 10.1179/joc.2004.16.2.119.

Bhatia, S., and Bharti, A. 2015. Evaluating the antimicrobial activity of nisin, lysozyme and ethylenediaminetetraacetate incorporated in starch based active food packaging film. Journal of Food Science and Technology 52(6): 3504-3512. doi: 10.1007/s13197-014-1414-7.

Carroll, K.C., and Bartlett, J.G. 2011. Biology of Clostridium difficile: implications for epidemiology and diagnosis. Annual review of microbiology 65: 501-521. doi: 10.1146/annurevmicro-090110-102824.

Chai, C., Lee, K.-S., and Oh, S.-W. 2015. Synergistic inhibition of Clostridium difficile with nisin-lysozyme combination treatment. Anaerobe 34: 24-26. doi:

http://dx.doi.org/10.1016/j.anaerobe.2015.04.003.

Chung, W., and Hancock, R.E.W. 2000. Action of lysozyme and nisin mixtures against lactic acid bacteria. International Journal of Food Microbiology 60(1): 25-32. doi:

http://dx.doi.org/10.1016/S0168-1605(00)00330-5.

Ghose, C. 2013. Clostridium difficile infection in the twenty-first century. Emerging Microbes and Infections 2: e62. doi: 10.1038/emi.2013.62.

Howerton, A., Ramirez, N., and Abel-Santos, E. 2011. Mapping interactions between germinants and Clostridium difficile spores. Journal of Bacteriology 193(1): 274-282. doi:

10.1128/JB.00980-10.

Kirby, A.J. 2001. The lysozyme mechanism sorted-after 50 years. Nature Structural \& Molecular Biology 8(9): 737-739. doi: 10.1038/nsb0901-737. 
Lucera, A., Costa, C., Conte, A., and Del Nobile, M.A. 2012. Food applications of natural antimicrobial compounds. Frontiers in Microbiology 3: 287. doi: 10.3389/fmicb.2012.00287. Nerandzic, M.M., and Donskey, C.J. 2013. Activate to eradicate: inhibition of Clostridium difficile spore outgrowth by the synergistic effects of osmotic activation and nisin. PLoS ONE 8(1): e54740. doi: 10.1371/journal.pone.0054740.

Permpoonpattana, P., Tolls, E.H., Nadem, R., Tan, S., Brisson, A., and Cutting, S.M. 2011. Surface layers of Clostridium difficile endospores. Journal of Bacteriology 193(23): 6461-6470. doi: $10.1128 / \mathrm{jb} .05182-11$.

Poutanen, S.M., and Simor, A.E. 2004. Clostridium difficile-associated diarrhea in adults. Can. Med. Assoc. J. 171(1): 51-58. doi: 10.1503/cmaj.1031189.

Preston, R.A., and Douthit, H.A. 1984. Stimulation of germination of unactivated Bacillus cereus spores by ammonia. Microbiology 130(5): 1041-1050. doi: doi:10.1099/00221287-130-5-1041. Ramirez, N., Liggins, M., and Abel-Santos, E. 2010. Kinetic evidence for the presence of putative germination receptors in Clostridium difficile spores. Journal of Bacteriology 192(16): 4215-4222. doi: 10.1128/jb.00488-10.

Sorg, J.A., and Sonenshein, A.L. 2008. Bile salts and glycine as cogerminants for Clostridium difficile spores. Journal of Bacteriology 190(7): 2505-2512. doi: 10.1128/jb.01765-07.

Theriot, C.M., and Young, V.B. 2015. Interactions between the gastrointestinal microbiome and Clostridium difficile. Annual Review Microbiology 69: 445-461. doi: 10.1146/annurev-micro091014-104115.

United States. Food and Drug Administration. 2016. Direct food substances affirmed as generally recognized as safe, 21 CFR 184. Washington, DC. 
Weber, D.J., Rutala, W.A., Miller, M.B., Huslage, K., and Sickbert-Bennett, E. 2010. Role of hospital surfaces in the transmission of emerging health care-associated pathogens: Norovirus, Clostridium difficile, and Acinetobacter species. American journal of infection control 38(5): S25-S33. doi: http://dx.doi.org/10.1016/j.ajic.2010.04.196.

Wilson, K.H. 1983. Efficiency of various bile salt preparations for stimulation of Clostridium difficile spore germination. Journal of Clinical Microbiology 18(4): 1017-1019. 


\section{Figure legends}

Fig. 1. The relative level of germinated and outgrowing $C$. difficile spores $(R L O)$ after $2 \mathrm{~h}$ of incubation in solutions containing: (A) $1 \% \mathrm{w} / \mathrm{v}$ sodium taurocholate alone (control), $1 \% \mathrm{w} / \mathrm{v}$ sodium taurocholate and peptone, yeast extract, tryptone, or tryptose $(1 \% \mathrm{w} / \mathrm{v})$; (B) $1 \% \mathrm{w} / \mathrm{v}$ sodium taurocholate and tryptose $(0,0.2,0.4,0.6,0.8$, or $1.0 \% \mathrm{w} / \mathrm{v}) ;(\mathrm{C}) 1 \% \mathrm{w} / \mathrm{v}$ sodium taurocholate, tryptose $(0.1 \% \mathrm{w} / \mathrm{v})$, and $\mathrm{NaCl}(0,0.25,0.5,1,5,10$, or $15 \% \mathrm{w} / \mathrm{v})$; or (D) $1 \% \mathrm{w} / \mathrm{v}$ sodium taurocholate, tryptose $(0,0.2,0.4,0.6,0.8$, or $1.0 \% \mathrm{w} / \mathrm{v})$, and $\mathrm{NaCl}(1 \% \mathrm{w} / \mathrm{v})$. The $R L O($ $R L O=L O / \log N_{0}$ ) was calculated using the level of germinated and outgrowing C. difficile spores $\left(L O ; L O=\log N_{0}-\log N_{2 h}\right)$ and the numbers of $C$. difficile spores before $\left(N_{0}\right)$ and after 2 h of incubation $\left(N_{2 h}\right)$. Data represented by the same letter are not significantly different at $\mathrm{P}<$ 0.05 .

Fig. 2. The relative level of surviving $C$. difficile spore $(R L S)$ during incubation in GO solution containing: (A) nisin $(0,20,40,60,80$, or $100 \mathrm{nM})$; (B) lysozyme $(0,0.2,0.4,0.6,0.8$, or 1.0 $\mathrm{mM})$; and $(\mathrm{C})$ both nisin $(0,5,10,15$, or $20 \mathrm{nM})$ and lysozyme $(0.2 \mathrm{mM})$. GO solution was composed of sodium taurocholate $(1 \% \mathrm{w} / \mathrm{v})$, tryptose $(1 \% \mathrm{w} / \mathrm{v})$, and $\mathrm{NaCl}(1 \% \mathrm{w} / \mathrm{v})$. The $R L S($ $\left.R L S=\log N_{t}^{s} / \log N_{0}^{s}\right)$ was calculated using the number of $C$. difficile spores at $0 \min \left(N_{0}^{s}\right)$ and a certain time $\left(N_{t}^{s}\right)$. 
(A)

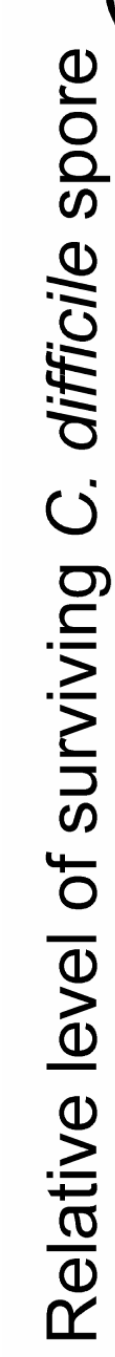

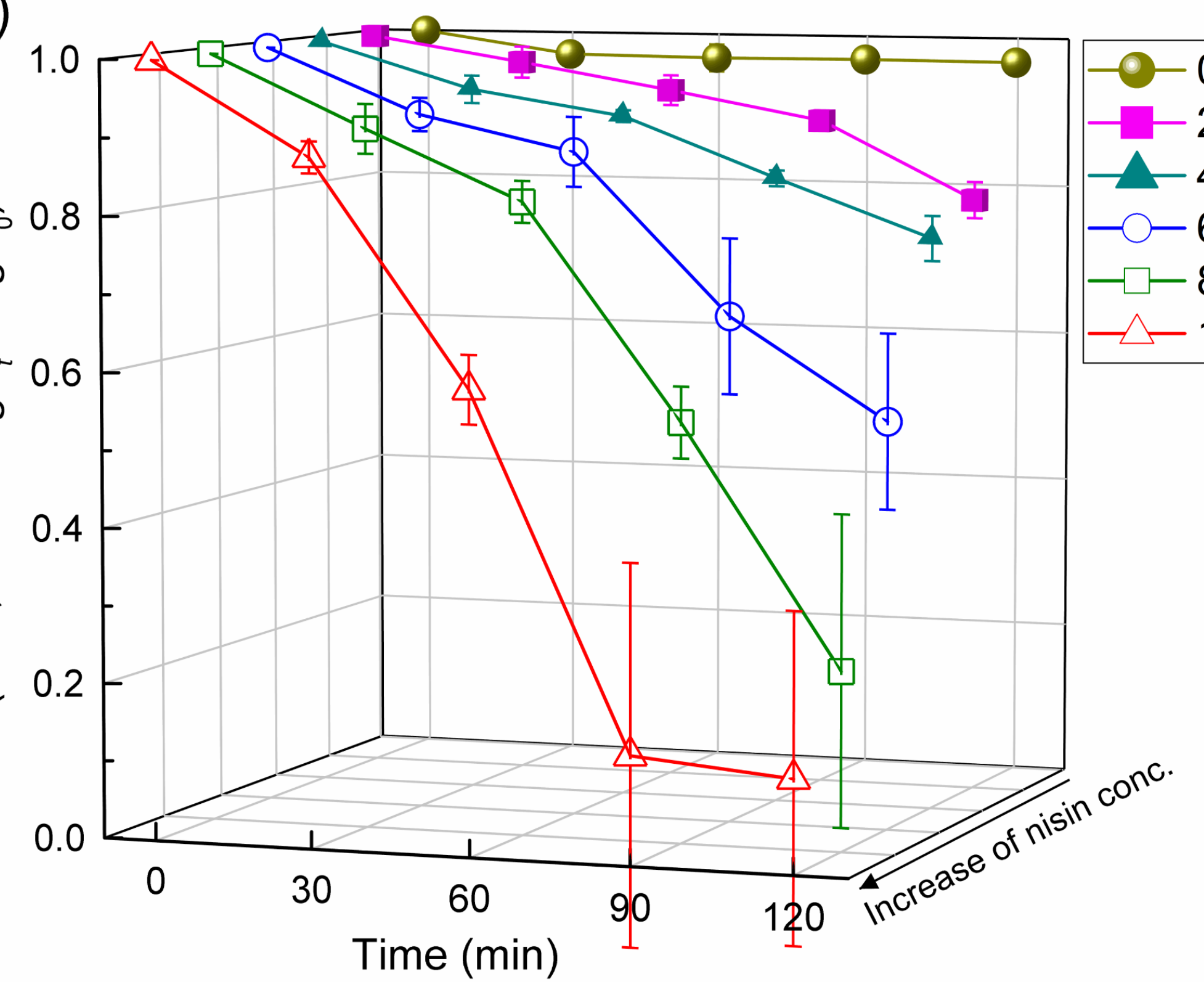

(B)

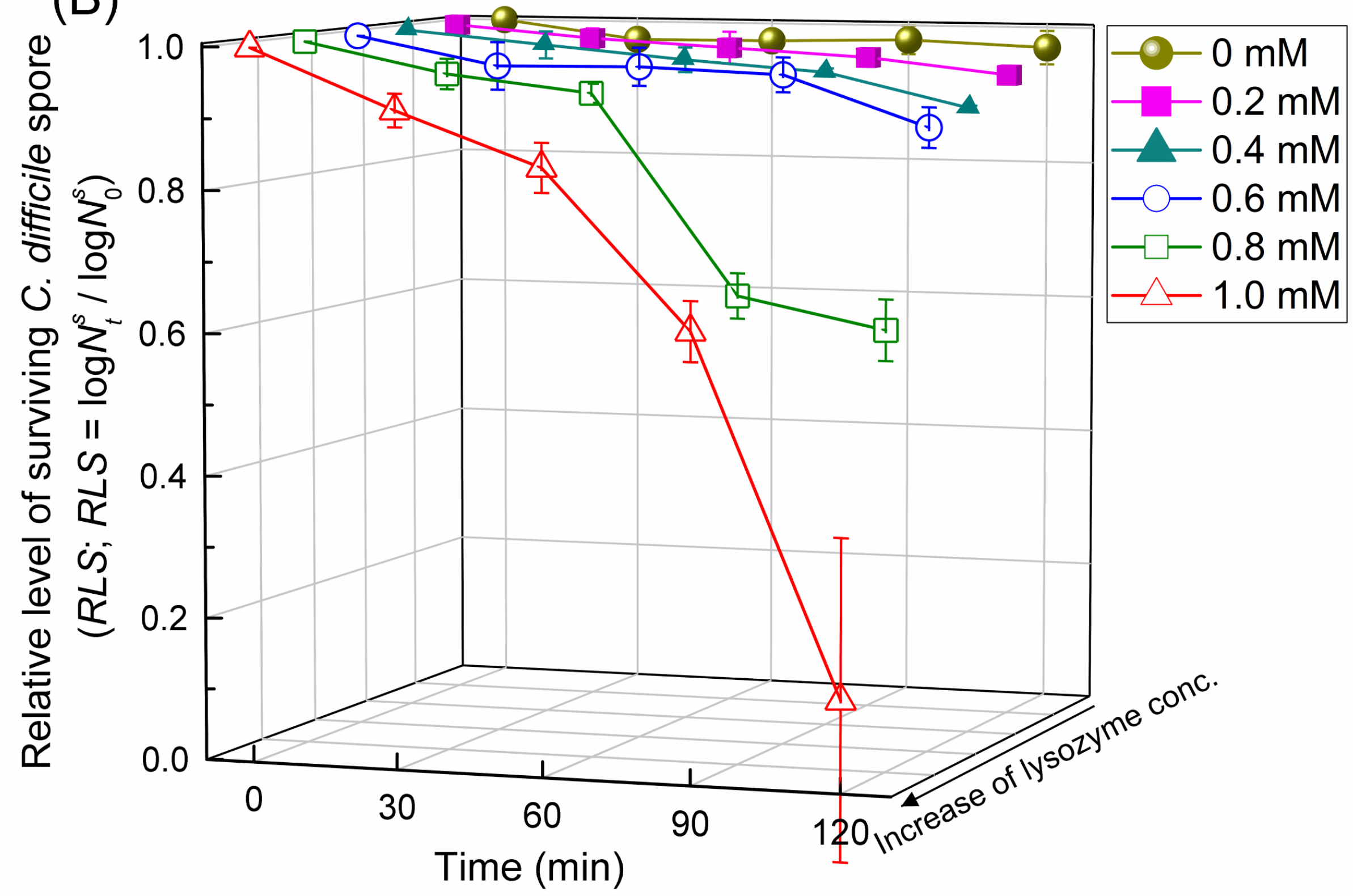

(C)

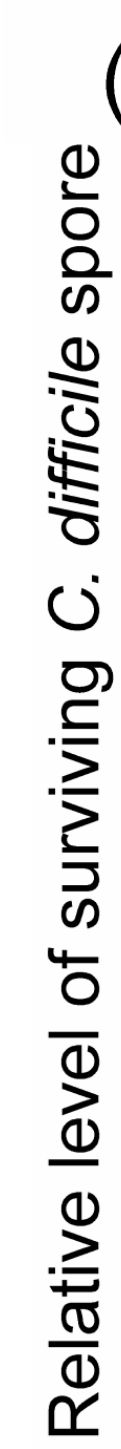

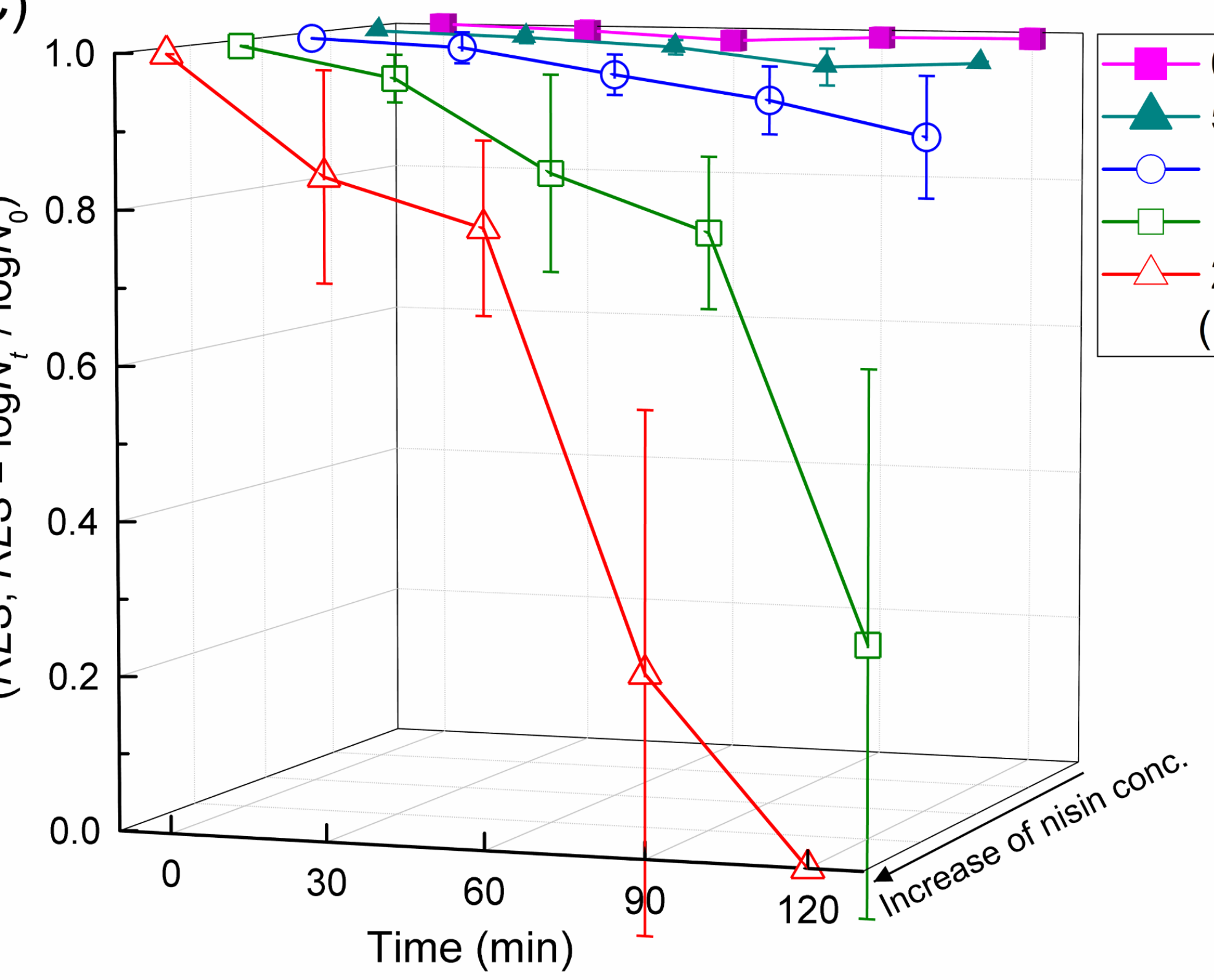

